\title{
Autonomous safety system for MSW landfills
}

\author{
Aleksandr Titov ${ }^{1, *}$, Aleksandr Tumanov ${ }^{1}$, Andrey Timofeev ${ }^{2}$, Vladimir Tumanov ${ }^{1}$, and Victor Denisov ${ }^{3}$ \\ ${ }^{1}$ Peter the Great St.Petersburg Polytechnic University (SPbPU), St. Petersburg, Russia \\ ${ }^{2}$ LLP "EqualiZoom", Astana, Kazakhstan \\ ${ }^{3}$ Flagman Geo Ltd, St. Petersburg, Russia
}

\begin{abstract}
The current state of landfills is an important issue for monitoring. There are a lot of municipal solid waste landfills in the world. But there are no standard solutions for monitoring the current state of these facilities both in Russia and abroad. It makes developing of technical solution, based on Autonomous sensors for measuring the concentrations of hazardous fumes, radiation background, geotechnical factors and other environmental variables, actual. Such system can be easily installed and can work in stand-alone mode for a long time. Decision-making technology in such system can be based on using of modern achievements of Machine Learning.
\end{abstract}

\section{Introduction}

Nowadays the issues of ecological using of resources of big cities and human's influ-ence on the environment are discussed widely, e.g. (Sergeev, etc, 2019) [1], (Didenko, etc, 2017) [2]. The task of monitoring the status of municipal solid waste (MSW) dumps and landfills has become of acute importance in the Russian Federation. Hundreds of thousands of legal and illegal dumps and landfills are registered in the country. At the same time, there are no standard solutions for monitoring the state of these facilities both in Russia and abroad. The situation is aggravated by the fact that each object has a unique form and a unique list of hazards. Therefore, It is proposed to develop a technical solution on the basis of autonomous sensors for measuring the concentration of hazardous fumes, radiation background, geotechnical factors. It is necessary to form a list of parameters of the landfill, which are the objects to constant monitoring. Each sensor must be equipped with a battery and communication module. This will allow you to create a monitoring system, configurable by both the composition of the sensors and the dimensions. Such a system can be easily installed at any site and will be able to work offline for several years, transferring data to remote control centres.

\section{Background}

Studies of the current state of the problem of ensuring environmental safety of land-fills showed that the effective use of economic instruments of environmental man-agement in the activities of landfills has been constrained by the lack of well-developed approaches to evaluation the risk of adverse effects of these objects on the environmental components $[3,4]$, unlike other risks in the field of environmental management, for which currently there is a detailed scientific and methodological apparatus, e.g. (Kotlyarov, Petrov, 2014) [5], (Borisovich, etc, 2018) [6].

Landfills, like any industrial enterprise, have limits of permissible emis-sions/discharges, limits on waste disposal and for a certain fee perform a set of measures for waste management, including reception and placement for sorting, processing and disposal. In the vast majority landfills arose spontaneously, without regard to environmental requirements, in waste pits, other types of pits, etc. The average square of each landfill is significant - from several tens to 120 hectares. The time resource of a landfill is developed in three to four years, while the amount of waste does not decrease from year to year, but, on the contrary, increases.

As a rule, to estimate the probability of damage resulting from the occurrence of environmental risks at the landfill, and hence the total cost of losses, several parame-ters are measured, such as the square of the landfill, the year of its completion and the limit of waste disposal.

However, these works do not consider the possibility of implementing technical systems for early warning of environmental risks. The results of their hazard assessment can play an important role in the management of municipal solid waste and in the development of an economic environmental management mechanism that can solve the problem of environmental pollution in the operation of municipal solid waste landfills.

Environmental risk is the probability of negative changes in the environment under the influence of adverse effects on the environment [4]. The following significant environmental risks inherent in the field of solid waste management can be identified:

1. Air pollution as a result of MSW landfill ignition. Fire occurs with a sufficient amount of oxygen in the

* Corresponding author: titov ab@spbstu.ru 
thickness of the landfill, when in addition to the oxidation of organic components, the oxidation of inorganic compounds occurs. Biochemical decomposition increases the temperature of the waste to 40-70 ${ }^{\circ} \mathrm{C}$, which activates the processes of chemical oxidation and leads to a further increase of temperature. As a result of biochemical processes in landfill soils, biogas is formed, which when it reaches the surface pollutes the atmospheric air and is easily flammable. Also, crisis (emergency) situations can arise in case of violation of fire-prevention requirements at reception of waste (delivery of burning garbage) [7,8].

2 . Ground pollution by heavy metals. Due to the lack of separate waste collection, as well as the imperfection of the technology of manual sorting, there is a high proba-bility of heavy metals entering the soil through landfills and unauthorized dumps.

3. Groundwater pollution in landfill sites. This is due to the lack of systematic con-trol by the supervisory authorities, the toxicity of waste, the release of filtrate in the process of rotting waste, precipitation and passage through the layers of waste, the lack of proper sealing of landfills [9].

4. Formation and emission of landfill gases into the atmosphere. Against the back-ground of periodically occurring prolonged inversions in the surface layer of the at-mosphere, local pollution occurs with increased concentrations of harmful substances, which are dangerous for people living near $[10,11,12,13]$.

5. Unrecultivated waste landfills. Decommissioned non-recultivated waste disposal facilities cause significantly higher accumulated damage to the environment. As a rule, there are not enough funds for the design of their recultivation and recultivation itself at local and regional budgets, given the need to simultaneously Finance the construction of a new solid waste disposal facility.

6. High probability of occurrence of infectious diseases centre at the waste disposal facilities.

As a rule, in this context the main principles of prevention of irreversible conse-quences for the environment are the following:

1. The correct choice of location for the polygons.

Polygons are placed outside the settlements in compliance with the size of the sani-tary protection zone established by normative documents.

2. Creation the technical design of landfills that prevent the penetration of pollu-tants into the components of the environment.

3. Proper operation of landfills. In the process of filling the landfill waste should be provided possibility for garbage trucks and construction equipment, as well as the overall stability of the construction of landfill soils. It is forbidden to accept certain types of waste to landfills.

4. Rationing in the field of waste management. In order to ensure the protection of the environment and human health, to reduce the amount of waste in relation to enterprises as a result of economic and other activities of which waste is generated, standards for waste generation and limits for their disposal are established.

5. Reducing the number of unauthorized landfills.
6. Quality control of stored waste and monitoring of the environment should be or-ganized.

The monitoring system is an information basis for determining the effectiveness of environmental measures, as well as a database for the development of technical and technological solutions to improve landfill operations $[14,15]$.

Monitoring programs can monitor:

- chemical composition and amount of filtrate formed in the landfill body;

- changes in the quality of groundwater outside the landfill (groundwater monitor-ing at landfills is carried out with the help of wells);

- air pollution, both in the working area on the territory of the landfill and beyond;

- compliance of the waste entering the landfill with the declared degree of danger [4].

The last one deserves the special attention.

With the active development of automatic monitoring in the last decades, new opportunities for intelligent collection and analysis of information about the state of landfills are appeared. For these purposes, subsystems of the lower level, which col-lect data can be used. And the middle and upper levels allow to assess, analyse and predict the development of adverse events in complex systems. Last times the Indus-try 4.0 have penetrated into the most various spheres of our life, e.g. (Glukhov, etc, 2016) [16], (Borisoglebskaya, etc, 2019) [17].

\section{Methods}

It is proposed to use a complex system of monitoring of landfills in order to minimize environmental risks. The main objectives of the integrated monitoring of polygons are the following:

- timely detection of exceedance of critical levels of hazardous fumes of methane $(\mathrm{CH} 4)$, carbon dioxide (CO2), and methanol, petrol, toluene and ethanol;

- control of internal deformation of the landfill, such as landslides and subsidence; - control of radiation situation on its territory;

- perimeter control;

- working in stand-alone mode (from internal power sources) and transfer of in-formation via wireless communication lines to the data collection Center in accord-ance with the adopted regulations;

- construction of a dynamic map of dangerous incidents in the area of the landfill, which is modified as the next data from the spatially distributed data collection system.

As a result a detailed database of recorded events should be maintained.

Figure 1 shows the block diagram of the integrated monitoring system of landfill. 


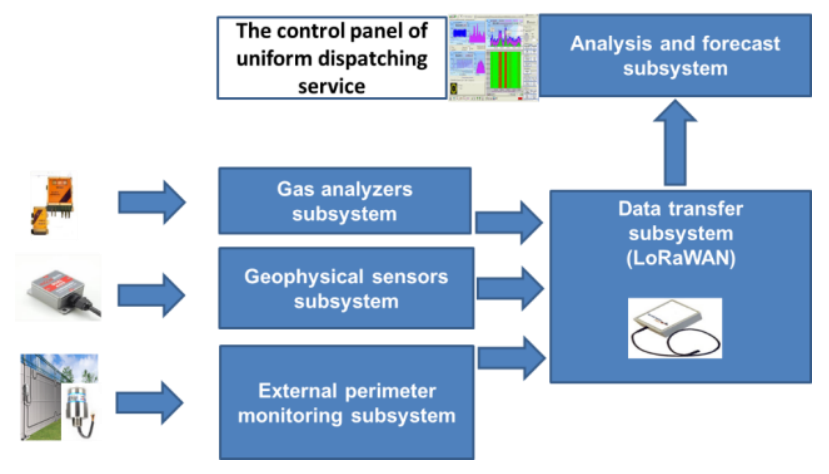

Fig. 1. Structure of the proposed landfill monitoring system.

It is necessary to consider each of the subsystems separately.

1. Gas analysers subsystem is a spatially distributed network of gas analyzers of various types, capable of detecting dangerous vapours from the target list with high reliability.

The location of the network sensors is determined by the structure of the polygon and its geotechnical parameters. Each sensor network is integrated with LoRaWAN modem for data transmission and to control its functioning [18]. The form factor of each sensor must ensure the normal operation of the sensor in severe weather condi-tions. The energy supply of the sensors is supplied from an independent power sup-ply.

2. The subsystem of geophysical sensors is a spatially distributed network of seis-mic sensors inclinometers, designed to monitor the state of the grounds of landfill and control their internal deformations. Inclinometric complex of soil control provides monitoring of soil movements and allows to assess the condition of the foundations of engineering structures, dams, quarries and other structures. The equipment of the complex provides a stationary installation, automatic operation and wireless data transmission during the entire calibration period. Energy supply of sensors is provided by batteries, fuel cells or combined power plants. The subsystem of geotechnical sensors can be equipped with additional systems for building monitoring and earthquakes monitoring.

3. External perimeter monitoring subsystem is designed to monitor activities in the area of the geometric boundaries of the landfill. It consists of a seismosensitive C-OTDR system [19], designed to monitor seismic activity in the area of the controlled perimeter, as well as a network of long-range surveillance cameras. This subsystem is a typical bimodal perimeter control system (modes: "video" and "seismic"). The video mode is provided by the use of long-range video monitoring systems (up to $3 \mathrm{~km}$ ), which are placed on special towers and are powered from the Central power plant or from the Autonomous power subsystem (diesel generator and solar panels). Seismic fashion is achieved by using C-OTDR system vibrosensors type. A sensitive sensor of this system is a standard optical fiber SMF-28, buried along the perimeter of the land-fill to a depth of $30-50 \mathrm{~cm}$. This system provides detection of a pedestrian at a dis-tance of 5 meters from the sensor when the value of the spatial resolution along the cable length from 5 to $10 \mathrm{~m}$. One analyser system capable of servicing a sensor length of up to $40 \mathrm{~km}$, while ensuring from 4000 to 8000 channels. Such systems have a reputation for being very reliable and relatively inexpensive solutions optimized for perimeter control of extended facilities [19].

4. Data transfer subsystem collects information from sensors of different types, the data transmission based on wireless technologies (LoRaWAN). Each sensor of gas analyzers and geotechnical sensors subsystems is equipped with LoRaWAN modem operating in unlicensed $868 \mathrm{MHz}$ range. Depending on the mode of operation of the monitoring system, each sensor is assigned an individual mode of operation, which depends on the type of sensor, the season, the situation at the monitoring site, etc. In accordance with the concept of LoRaWAN, each modem is equipped with a battery that guarantees up to 8 years of operation of the modem without recharging the bat-tery. Data from LoRaWAN modems is collected at LoRaWAN Base Stations. The number of these stations depends on the square of the landfill, one Base Station is able to serve up to 5000 modems at a distance of 3-5 km. Each Base Station is equipped with a conventional GSM-modem, which is connected via a mobile network type $2 \mathrm{G}$ (or higher) with a mobile operator to access the Internet via a secure channel. If the location of the landfill is not mobile or is it unstable, connection LoRaWAN Base Station with mobile operators can operate under a special radio channels with encrypted traffic. This is a relatively inexpensive and very effective solution. The proposed topology of this network is "star".

5.Analysis and forecast subsystem of intellectual analysis and prediction of the landfill state dynamics is based on complex accounting of information about the geological state of the polygon body, a network of precedent events of emission of target gases and spatiotemporal dynamics of the event flow.

This subsystem is designed to solve the short-term predictive problem of the land-fill state on the basis of a set of data collected from subsystems of gas analyzers and geotechnical sensors, as well as initial data on the geophysical structure of the landfill.

Decision-making technology is based on using of modern achievements of Ma-chine Learning [20].

Figure 2 represents a general scheme of the Analysis and forecast subsystem. 


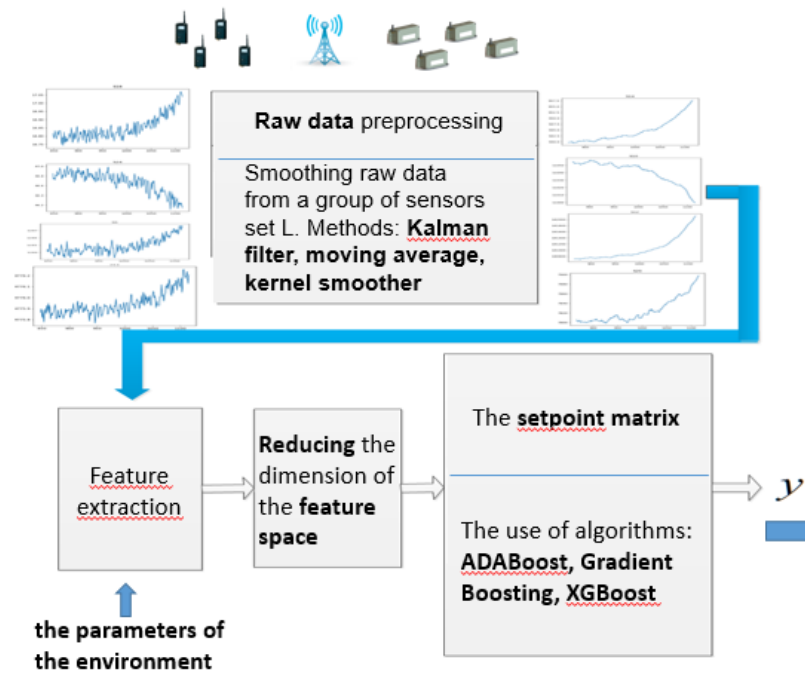

Fig. 2. General scheme of the Analysis and forecast subsystem.

Conditions for realization of Data Driven Approach are represented by operational control of the landfill, particularly control of the current parameters values, the set-point matrix (a combination of the passport of monitoring, which describes the boundary values of integral characteristics).

The setpoint matrix is based on the empirical model of environmental safety of the polygon. The setpoint matrix can be represented as a number of "alert levels".

Then It is necessary to enter the parameters of the external environment: wind speed, temperature, pressure, humidity, etc.

So we will have the condition for generating historical data: a combination of pa-rametersm which causes a precedent (dataset).

Setpoint matrix can be operatively changed (if necessary).

At the first stage, raw data received from sensors is preprocessed and smoothed (by using standart methods).Next stages are generating the feature space of the object and reducing its dimension.It is important to note that the model should contain as many environmental variables as possible.Then a comparison with the setpoint matrix and identification of patterns using a group of mathematical models and AI tools should be held. (Modern boosting methods of classification and regression analysis (ADABoost, Gradient Boosting, XGBoost).

As part of this approach, a predictive system, based on historical observations for each dataset, forms a forecast estimate of period of time before the incident will occur (explosive release, collapse, etc.)

Version of the forecast can be the following: "under the current dynamics of the concentration of harmful substances, the weather forecast, as well as environmental parameters, there are approximately 12 hours left before a dangerous incident»

Thus, for this research forecasting is understood as a probabilistic assessment of the time period before an incident will occur at the certain NSW landfill.

\section{Discussion and results analysis}

It is necessary to emphasize that it makes no sense to compare the results of this research with alternative studies in the field of landfill management. We are not considering options for incineration of waste or complete recycling of waste after sorting - we are considering building an effective system for preventing emergencies at existing landfills.

Figure 3 shows an illustration of the implemented system superimposed on the map of the existing landfill.

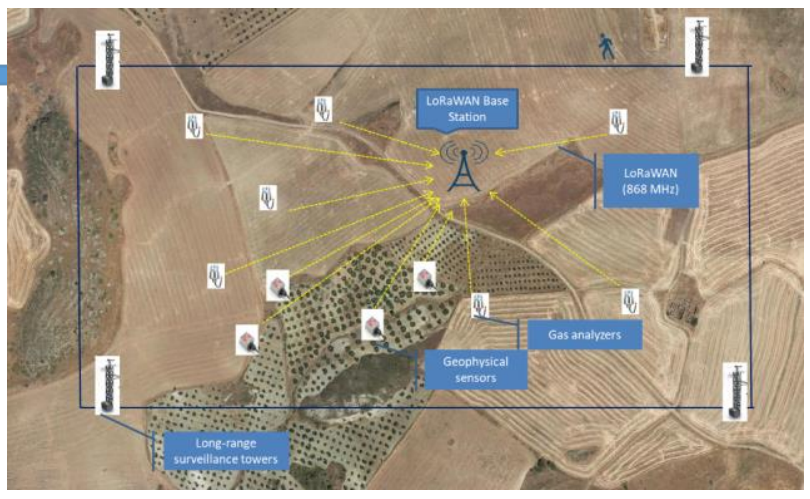

Fig. 3. An example of system implementation.

Implementation and using of autonomous safety system for MSW landfills requires an investment and operation costs.

The integral efficiency should reflect the synergetic effect of obtaining new oppor-tunities for management decisions at the certain landfill. These management decisions can potentially reduce or even eliminate the costs of liquidation of consequences of emergency situations, investigative measures, repairs, etc.

It is necessary to correlate investment and operation costs on one side with the general savings from another side in order to estimate economic efficiency of creation the offered monitoring system on the certain MSW landfill.

According to opinion of many researches, the use of the proposed technology has a high potential in the Eurasian Economic Union (EAEU) [21].

\section{Conclusion}

Nowadays the proposed monitoring system for does not have effective serial solutions both in Russia and abroad.

The proposed concept can be implemented as in Russia as abroad. It will be a net-work of autonomous wireless sensors capable of transmitting information in the LoRa format to its own Base Station, which will transmit the received data via mobile communication channels at any distance to the Control Centre.

The maximum distance from the sensors to the base station is not more than $15 \mathrm{~km}$. The frequency of the survey - at least once an hour. Battery life without battery replacement should be up to 5 years.

The use of wireless technologies will allow to reconfigure the network of sensors for objects of different size and shape. 
The composition of the sensors used can easily vary depending on the conditions of a particular landfill.

The integral efficiency reflects the synergetic effect of reducing and elimination the costs of liquidation of consequences of emergency situations, investigative measures, repairs, etc.

This paper was financially supported by the Ministry of Education and Science of the Russian Federation on the programme to improve the competitiveness of Peter the Great St.Petersburg Polytechnic University (SPbPU) among the world's leading research and education centres in the 20162020 .

\section{References}

[1] S. Sergeev, T. Kirillova, I. Krasyuk, Modelling of sustainable development of megacities under limited resources, E3S Web of Conferences, 91, 05007 (2019).

[2] N. Didenko, D. Skripnuk, O. Mirolyubova, The effects of human behavior on fresh water resources, International Multidisciplinary Scientific, GeoConference, Surveying Geology and Mining Ecology Management, SGEM, 17, 53, 901-910 (2017).

[3] A. Mudretsov, A. Tulupov, Estimation of ecological risks of landfills, Regional problems of transformation of the economy, 3, 37, 242-247 (2013).

[4] E. Derkacheva, N. Razinkov, Emergency situations related to the fire of solid waste disposal sites and the way to solve the problem, Complex problems of technosphere safety: materials international. scientific. Conf., 12 November 2015, Voronezh, part 9, 138-142 (2016).

[5] I. Kotlyarov, S. Petrov, Risk assessment procedure for economic-geological and cost estimate of mineral deposits, Gornyi Zhurnal, 9, 94-99 (2014).

[6] V. Borisovich, N. Kurbanov, V. Zaernyuk, B. Sefullaev, Practical risk management at gold mining companies, Gorny Zhurnal, 11, 70-75 (2018).

[7] M. Vaverková, D. Adamcová, Long-Term Temperature Monitoring of a Municipal Solid Waste Landfill, Polish J. Environ. Stud., 24, 13731378 (2015).

[8] J. Shi, T. Zhang, J. Zhang, Y. Ai, Y. Zhang, Prototype heat exchange and monitoring system at a municipal solid waste landfill in China, Waste Manag., 78, 659-668 (2018).

[9] D.D. Lopes, et al., Geophysical technique and groundwater monitoring to detect leachate contamination in the surrounding area of a landfill - Londrina (PR - Brazil), J. Environ. Manage, 113, 481-487 (2012).

[10] D. Jovanov, B. Vujić, G. Vujić, Optimization of the monitoring of landfill gas and leachate in closed methanogenic landfills, J. Environ. Manage, 216, 32-40 (2018).
[11] R. Beaven, C. Scheutz, Landfill gas emission monitoring, Waste Manag., 87, 833-834 (2019).

[12] T. Kormi, S. Mhadhebi, N. Bel Hadj Ali, T. Abichou, R. Green, Estimation of fugitive landfill methane emissions using surface emission monitoring and Genetic Algorithms optimization, Waste Manag., 72, 313-328 (2018).

[13] Z. Xing, et al., Real-time monitoring of methane oxidation in a simulated landfill cover soil and MiSeq pyrosequencing analysis of the related bacterial community structure, Waste Manag, 68, 369-377 (2017).

[14] C. Iacoboaea, F. Petrescu, Landfill monitoring using remote sensing: a case study of Glina, Romania, Waste Manag. Res., 31, 1075-1080 (2013).

[15] G.F. Simões, C.A.A.Catapreta, Monitoring and modeling of long-term settlements of an experimental landfill in Brazil, Waste Manag., 33, 420-430 (2013).

[16] V. Glukhov, I. Ilin, O. Iliashenko, Improving the efficiency of architectural solutions based on cloud services integration Lecture Notes in Computer Science (including subseries Lecture Notes in Artificial Intelligence and Lecture Notes in Bioinformatics), 9870 LNCS, 512-524 (2016).

[17] L. Borisoglebskaya, E. Provotorova, S. Sergeev, A. Khudyakov, Automated storage and retrieval system for Industry 4.0 concept, International Scientific Workshop "Advanced Technologies in Material Science, Mechanical and Automation Engineering", MIP: Engineering-2019, IOP Conf. Ser.: Mater. Sci. Eng., 537, 032036 (2019).

[18] O. Gusev, Experiment to create a monitoring system of economic objects using LORAWAN, Wireless technology, 2, 43, 72-76 (2016).

[19] A. Timofeev, Comparison of various approaches to multi-channel information fusion in C-OTDR systems for remote monitoring of extended objects, Scientific and technical Journal of information technologies, mechanics and optics, 15, 1, 122-129 (2015).

[20] P. Flah, Machine learning, DMK Press, Moscow (2015).

[21] L. Progunova, T. Voronova, S. Bogatyreva, O. Kostyukova, Innovative aspects of preferential rules of goods origin in the economy of global chains: applicability for the Eurasian Economic Union (EAEU), IOP Conference Series: Materials Science and Engineering 2019, 012048 (2019). 